\title{
The effects of chewing gum on perceived stress and wellbeing in students under a high and low workload
}

\author{
Andrew P Smith ${ }^{1}$ and Hope Clayton, ${ }^{1}$ \\ ${ }^{1}$ Centre for Occupational and Health Psychology, School of Psychology, Cardiff University, \\ 63 Park Place, Cardiff CF10 3AS, \\ smithap@cardiff.ac.uk
}

\begin{abstract}
Research has suggested that chewing gum relieves perceptions of stress in stressed and nonstressed individuals, but is most beneficial for those experiencing a greater amount of negative work characteristics. To determine if this was true in a student sample, the present study assigned 36 students to four one-day intervention conditions in which participants either chewed or refrained from gum and experienced a high or low workload. The results showed that an individual's perceptions of stress and mental fatigue decreased as a result of chewing gum. Depression and cognitive failures decreased as a result of experiencing a high workload. Perceptions of physical fatigue decreased when chewing gum under a high workload. The findings suggested that gum may be an effective way to reduce certain stress characteristics, and also reassuring students that a high workload is not necessarily detrimental to their wellbeing.
\end{abstract}

Keywords: workload; chewing gum; wellbeing

\section{Introduction}

\subsection{Mental Workload}

Mental workload has been widely studied [1,2] over a long period of time [3 4]. The research has been carried out in both laboratory [5, 6] and occupational settings [7, 8], and a variety of measures of workload have been developed [9, 10, 11, 12, 13, 14]. Workload measures include self-assessment, task measures and physiological measures. Self-assessment measures have taken several forms such as the NASA Task Load Index [15], the Workload Profile [16] and the Subjective Workload Assessment Technique [1]. Recent research has confirmed that even single items about perceptions of workload can predict the wellbeing of workers and students. Other approaches have examined specific components of workload, such as time pressure, and one example of this is the Karasek Job Demands scale, which predicts health and safety outcomes of workers [17]. Overall, there has been increased recent interest in both models and applications on research on mental workload [18, 19, 20].

University life can induce a prolonged period of stress as students face academic demands to succeed, competition with peers, financial strain, the social pressures and the usual daily issues that occur as a by-product of independence [21]. Studies have shown that acute, time-limited stress serves an adaptive function [22, 23, 24], whereas chronic ongoing stress is often detrimental to an individual's psychological and physical wellbeing [25]. For example, research using student samples has detected significant correlations between illness and stress, anxiety and depression [26]. The student population has risen by 44\% in recent years [27], and despite increases in tuition fees, the number of university applicants and places is not expected to decline. The historically-large numbers of individuals attending universities suggests that poor psychological and physical health in students is potentially more prevalent and of more concern than ever.

\subsection{Chewing gum and stress}

Research has shown that interventions designed to target stress in students can impact positively on their perceived stress levels, depressive symptoms, coping [28], self-esteem [29], academic success [30] and health [31]; however, the majority of these interventions are costly and time-consuming. According to the Association for University and College Counselling, universities are insufficiently equipped to provide services that facilitate these interventions [32], and due to government cuts in university funding, this problem is only going to increase. Chewing gum may be a relatively inexpensive and effortless way to reduce stress. In a survey involving 8,930 university students, $41 \%$ claimed to chew gum to relieve stress and tension [33], suggesting chewing gum may aid stress relief. As research has shown that students may engage in negative behaviors to 
relieve stress such as consuming alcohol [34], smoking [35] and illicit drug use [36], chewing gum also appears to be a healthier alternative. The beneficial effects of chewing gum were first documented when Hollingworth [37] demonstrated that collateral motor automatisms involving masticatory muscles can reduce self-reported and direct muscular tension, suggesting that chewing gum can operate as a form of relaxation. However, Freeman [38] consequently showed that foot-tapping was as effective as chewing gum in reducing tension, suggesting that the relaxing effects elicited by motor automatisms are not limited to chewing gum.

Research has also examined whether chewing gum can reduce naturally occurring stress [39]. A web-based survey of 2,248 full-time workers found that despite reporting a higher prevalence of negative occupational demands, gum chewers demonstrated lower levels of perceived stress and depression and were less likely to have seen their doctor for high blood pressure or cholesterol compared to non-gum chewers [40]. These results remained after controlling for health-related behaviors, personality, negative job characteristics and a range of demographics; however, due to the cross-sectional nature of the study it was not possible to establish whether gum does indeed reduce stress or if those who are less stressed have chosen to chew gum.

Intervention studies have helped to clarify the matter, suggesting that chewing gum exerts a positive effect on perceived stress. Zibell and Madansky [41] used a cross-over design to examine whether those who did and did not regularly chew gum would perceive themselves as less or more stressed after abstaining or chewing gum for a period of time and then subsequently switching to the opposite chewing behavior. The results demonstrated that during periods of abstinence, perceived stress levels increased significantly in those who did and did not regularly chew gum. During periods of chewing, the opposite pattern was observed; perceived stress was significantly reduced in both groups. These findings suggest that chewing gum aids stress reduction regardless of individuals' regular gum-chewing habit. However, the study was based on self-perception and asking participants to directly assess whether gum had reduced their levels of stress implies that demand characteristics were present [42]. It is therefore likely that the results, while interesting, cannot be considered conclusive and should be subject to intervention studies involving a more reliable methodology.

To address this, Smith, Chaplin and Wadsworth [43] used a cross over design to determine whether chewing gum for at least 20 minutes a day for two weeks would reduce work and life stress, fatigue, depression and cognitive failures and improve mood and work performance in university employees. The results demonstrated that chewing gum was associated with reduced stress, greater wellbeing and enhanced performance at work, suggesting that chewing gum over a relatively long time has beneficial effects. In another study involving university staff, participants were required to either abstain or chew gum for one workday [44]. Questionnaire measures completed at the beginning and end of the day demonstrated that chewing gum was associated with fewer cognitive problems, but had no significant effect on fatigue, occupational stress, anxiety, depression or being behind at work. Allen and Smith [44] suggest that the discrepancies between these two studies involving university employees may be due to the short nature of the intervention in their study; it is possible that in order to see beneficial effects of chewing gum on mental health and wellbeing, the gum must be chewed for an extended time period. A longer intervention was undertaken to investigate whether the findings of Smith et al. [40] could be replicated in a student sample. Smith and Woods [45] instructed participants to either partake or refrain from chewing gum for a period of two weeks. Their analysis demonstrated that those who had chewed gum considered themselves significantly less stressed post-treatment compared to those who had refrained from chewing gum. This effect occurred in a dose-response fashion so that those who chewed the most gum perceived themselves as the least stressed.

\subsection{The present study}

Research examining the effects of chewing gum on naturally-occurring stress in students has so far failed to address whether it exerts beneficial effects on all levels of stress. Chewing gum has been shown to exert positive effects on stressed and non-stressed individuals [46], and it appears that chewing gum is most beneficial to those experiencing a greater degree of negative work characteristics [40]. The present study aimed to determine causality by investigating whether the proposed benefits of chewing gum under a high workload would apply to a student sample. Although research has shown that chewing gum does enhance wellbeing in students, it has not examined the effects of workload on perceived stress. It is, therefore possible that all participants were experiencing a high degree of stress due to a heavy workload, accounting for the beneficial effects of chewing gum. To address these issues, a within-subjects intervention design was used here. Research has shown that chewing gum for 20 minutes a day is sufficient to exert beneficial effects on an individual's perceived stress and wellbeing [43, 45]. Participants were, therefore, instructed to do so under a high and low workload. A control condition was also included; participants refrained from chewing gum under a high and low workload. As previous research has suggested that a one-day intervention is sufficient to exert beneficial effects on characteristics of perceived stress and wellbeing [44], the four conditions each lasted a day. Given that chewing gum has been associated with a vast range of stress and wellbeing phenomena including stress, anxiety, depression, fatigue, cognitive failures, and the ability to get work done, this study assessed these variables. 
Participants reported their self-perceptions via an online questionnaire at the beginning of each day and at the end of each day. Given that the study was investigating perceived stress, instructing participants to complete several questions, each measuring a single construct may itself have affected stress levels. Single-item measures of wellbeing are highly correlated with their full-scale counterparts [47]. For example, single item questions relating to work characteristics, appraisals and outcomes were found to be an adequate replacement for the demands-control-support questionnaire, the revised ways of coping checklist, the hospital anxiety and depression scale, and the patient health questionnaire using a university employee sample [48]. As a significant effect of chewing gum on perceived stress has been demonstrated using these single-item questions with an occupational sample [44], the present study employed single-item measures to assess participants' self-reported perceptions of stress and wellbeing in the student sample.

\section{Method}

Two hypotheses were explored:

(1) Chewing gum has been shown to exert positive effects on a number of variables in both stressed and nonstressed individuals. The first hypothesis predicted a main effect of chewing gum; under both high and low workload, chewing gum would reduce stress, anxiety, depression, mental and physical fatigue, problems with memory, attention or action, and being behind at work compared to the no gum control conditions.

(2) Research has also demonstrated that chewing gum is most beneficial to those experiencing a high degree of negative work characteristics. The second hypothesis predicted an interaction between chewing gum and the severity of workload - participants would experience a greater reduction in the variables listed in hypothesis 1 when experiencing a high workload and chewing gum.

This study was carried out with the approval of the ethics committee, School of Psychology, Cardiff University and the informed consent of the volunteers.

\subsection{Design}

The experiment had two independent variables: degree of workload, and gum chewing. These were both withinsubject variables. Each IV had two levels: high and low workload, and chewing or refraining from gum. The dependent variables were the difference scores (end of working day - beginning of working day) for the measures of stress, depression, anxiety, mental and physical fatigue, problems with memory, attention or action and getting behind with work. The orders of gum conditions were counterbalanced and high and low workload conditions were counterbalanced within the gum conditions. Participants were randomly allocated to a particular order in which to complete the conditions.

\subsection{Materials: Instruction sheets}

Participants received one of eight instruction sheets, identical but for the heading at the top of the sheet, stipulating the order that the conditions were to be completed. The aim of the experiment was briefly described and the subsequent task outlined in numbered bullet points. Participants were to select two periods of six days within the next two weeks, and within each period were to choose one high workload day and one low workload day. On one of the high and low workload days they were to chew at least two pieces of gum for at least 20 minutes; on the other two days, they were to refrain from chewing gum. For undergraduate students, a high workload day was defined as a day in which participants had two or more lectures and were working on an assignment and/or in paid or voluntary work whilst a low workload day was defined as one during which participants had one or fewer lectures and/or were not working on an assignment or in paid or voluntary work. $\mathrm{PhD}$ students were able to replace the lecture criteria with research, so that one lecture corresponded to one hour of research work. The final point stated that participants would be e-mailed the link to the online survey, which they were to complete at the beginning and end of each of their four chosen days (see Appendix A for an example instruction sheet).

\subsection{Gum}

Participants were given two packets of chewing gum. To promote chewing, they were allowed free choice from a range of flavors from the Wrigley's sugar-free Extra and Extra Ice range (spearmint, peppermint, cool breeze). Participants recorded how much gum they had consumed at the end of each of the four days. 


\subsection{Online Questionnaire}

The questionnaire was written and accessed via Survey Tracker. Participants firstly indicated how many pieces of chewing gum they had consumed so far that day, how many hours of lectures they had attended and how many hours they had spent studying and/or in paid or voluntary work before completing questions taken from Allen and Smith's [41] one-day intervention study. Using a 7-point Likert scale, participants were asked to indicate how stressed, anxious, depressed and mentally and physically fatigued they felt at that time, and whether they were experiencing problems of memory, attention or action, or getting behind with work (see Appendix B).

\subsection{Procedure}

At recruitment participants were invited into the lab, two at a time. Each participant sat at a desk at opposite ends of the room so that they could not communicate. They were instructed to read and sign the consent form, advising that the study was investigating the effects of chewing gum on perceived stress. Participants were handed the instruction sheet and were asked to read it. The experimenter also read them aloud to each participant before asking if they had any questions regarding their subsequent task. It was explained that the instructions, survey link and their participant number would be e-mailed to them as soon as they left the lab. Participants were informed that they would be e-mailed the debrief form and granted their course credit when they had completed the study. Before leaving the lab participants were invited to take two packets of chewing gum. Throughout the task the experimenter kept in regular contact with the participants to avoid attrition; when participants had completed their first questionnaire of the day, a reminder e-mail was sent to remind them to complete the second questionnaire. Participants also received an e-mail one week after commencing the task, prompting them to continue participating and thanking them for their efforts. When participants had completed all their questionnaires they were e-mailed a debrief form.

\subsection{Analysis}

To control for individual differences, statistical tests were carried out on the difference scores of each DV. Difference scores were calculated by subtracting the beginning of the day score from the end of the day score for each variable in each condition for each participant. Therefore, a minus score reflected that participants were less stressed at the end of the day. To examine any main effects or interactions between the IVs, thereby investigating hypothesis 1 and 2, a two-way repeated- measures ANOVA was conducted for each DV.

\section{RESULTS}

The descriptive statistics for all variables are shown in Table 1.

Table 1: Mean difference scores in the workload and gum conditions.

\begin{tabular}{|c|c|c|c|c|}
\hline & $\begin{array}{c}\text { High } \\
\text { Workload } \\
\text { and Gum }\end{array}$ & $\begin{array}{l}\text { High } \\
\text { Workload } \\
\text { No Gum }\end{array}$ & $\begin{array}{c}\text { Low } \\
\text { Workload } \\
\text { Gum }\end{array}$ & $\begin{array}{c}\text { Low } \\
\text { Workload } \\
\text { No Gum }\end{array}$ \\
\hline Stress & -0.60 & 0.08 & -0.42 & 1.8 \\
\hline Mental Fatigue & -0.24 & 0.83 & 0.50 & 0.71 \\
\hline Depression & -0.24 & -0.31 & 0.06 & 1.40 \\
\hline $\begin{array}{l}\text { Problems of } \\
\text { memory, attention } \\
\text { and action }\end{array}$ & -0.47 & 0.05 & 0.43 & 0.31 \\
\hline Physical Fatigue & -0.45 & 0.90 & 0.25 & 0.52 \\
\hline $\begin{array}{l}\text { Getting behind with } \\
\text { work }\end{array}$ & -0.05 & 0.20 & 0.12 & 0.23 \\
\hline Anxiety & -0.60 & -0.48 & -0.53 & -0.18 \\
\hline
\end{tabular}

\subsection{Main effects of chewing gum}

There was a significant main effect of chewing gum on stress, $F(1,35)=4.73, p<.05$, MSE $=14.69$. The effect was in the expected direction, so that a significant reduction in stress occurred when chewing gum under both high and low workload, compared to refraining from chewing gum under a high and low workload. There was 
also a significant main effect of mental fatigue, $\mathrm{F}(1,35)=6.52, \mathrm{p}<.05, \mathrm{MSE}=12.25$. Participants reported significantly reduced mental fatigue when chewing gum under both high and low workload compared to refraining from gum under a high and low workload.

\subsection{Main effects of workload}

There was a significant main effect of workload on depression, $\mathrm{F}(1,35)=4.58, \mathrm{p}<.05, \mathrm{MSE}=5.062$. This occurred in the expected direction, so that participants considered themselves as significantly less depressed under a high workload compared to the low workload condition when chewing or refraining from gum. A significant main effect of workload on problems with memory, attention or action was also detected, $\mathrm{F}(1,35)=$ $5.46, \mathrm{p}<.05, \mathrm{MSE}=8.03$. Problems with memory, attention or action were significantly reduced when experiencing a high workload when chewing or refraining from gum compared to the low workload conditions.

\section{Interaction between workload and gum conditions}

The interaction between gum condition and workload for reported physical fatigue difference scores was significant, $\mathrm{F}(1,35)=4.81, \mathrm{p}<.05, \mathrm{MSE}=9.51$. A significant reduction in physical fatigue was found when participants chewed gum under a high workload compared to chewing gum under a low workload, and refraining from gum under a high and low workload.

\subsection{Non-significant effects}

There were no significant effects of gum or workload, either as main effects or interactions, for the anxiety or getting behind with work variables.

\section{Discussion}

\subsection{Effects of chewing gum}

The study set out to address whether chewing gum under a high and low workload would affect participant's perceptions of stress and wellbeing. The results from the present study partly supported the hypotheses. There was a main effect of gum on stress and mental fatigue so that those who chewed gum experienced the most beneficial effects; furthermore, this effect was enhanced under a high workload. Taken together, these results support hypotheses 1 and 2. A significant interaction was also detected for measures of physical fatigue so that participants experienced the greatest benefit when chewing gum under a high workload. The means demonstrated that perceptions of stress were lower in the gum conditions. Together, these findings also support hypotheses 1 and 2. The workload manipulation was also somewhat successful; under a high workload participants experienced a greater reduction in depression and problems with cognition, but contrary to hypothesis 1 , this occurred in both gum conditions. With respect to stress and mental fatigue, the findings corroborated results from previous research [37,40], demonstrating that gum had a significant beneficial effect on stress and mental fatigue in stressed and non-stressed students. Several studies suggest there are plausible biological mechanisms underlying these effects [34], although this type of research is still in its infancy. Chewing gum exerted main effects on stress and mental fatigue, but several stress variables previously shown to be reduced after chewing gum [43,45] did not appear to be affected. As Allen and Smith [44] suggest, this may have been because previous research used a longer intervention period. Chewing a minimum of two pieces of gum in a one-day intervention may not have been enough to find significant main effects of chewing gum on these variables.

\subsection{Effects of Workload}

A main effect of workload on some of these variables was detected instead; self- reported perceptions of depression and problems with memory, attention and action were significantly reduced in high workload conditions. While this does not completely corroborate previous research that found a greater association between chewing gum and negative work characteristics, finding an effect of workload with or without gum is interesting in itself. It may indicate that individuals are distracted from feelings of depression or problems with cognition. Indeed, research has demonstrated that distraction reduces the intensity of negative mood, which may exert a knock-on effect on an individual's perception of their cognitive abilities. Additionally, or alternatively, the reductions in these perceived stress scores by the end of the day might signify feelings of relief, or satisfaction, having anticipated a stressful experience at the beginning of the day. It is feasible that this 
expectation may have arisen from labelling the days as 'high' or 'low' workload days, consequently engendering expectations about participant's imminent experiences. However, as an effect of workload was not found for all variables, this possibility is unlikely. Failing to find an effect of workload on mental fatigue and stress is probably due to the mentally demanding nature of the workload manipulation. As research has shown physical activity to override the negative effects of psychological stress and mental fatigue, future research should examine whether differences in a physical workload affect these variables when chewing or refraining from gum.

\subsection{Interactions between Workload and Chewing Gum}

A significant interaction was also detected for measures of physical fatigue; participants experienced the greatest benefit when chewing gum under a high workload. The means fell in the expected direction; fatigue scores were lower in the gum conditions than the control conditions, corroborating previous research. There were no significant main effects or interactions found for being behind at work. Although the research with an occupational sample has found effects of chewing gum on this variable [40], getting-behind with work may arguably be perceived as more severe within a student sample given that the present sample were attending university, presumably with the main intention of studying. According to previous research, more severe types of stress are unaffected by chewing gum [41], which may account for the non-significant effects of gum on getting behind with work with this student sample. Alternatively, it may be that this variable is unlikely to be affected by a one-day intervention; getting-behind with work is often not apparent for several weeks.

\subsection{No effect of Workload or Chewing Gum}

Additionally, the analysis failed to find any main effects or interactions with respect to anxiety. Somewhat surprisingly, the average difference scores for the four conditions demonstrated that participants were considerably less anxious at the end of the day in all four conditions. Given that this trend was not found in any other variables, it is likely that this finding owes itself to the considerable variation in participant's responses. Future research should replicate the experiment, taking note of the suggestions for a tighter methodology, as mentioned in the discussion, to determine whether these individual differences can be reduced.

\subsection{Limitations and future research}

The present study had several limitations that may facilitate an understanding of why the experiment failed to fully support the hypotheses. Firstly, the dependent variables relied on a participant's accurate and consistent recognition of perceived stress. Although

the questionnaires were designed with convenience in mind, it is likely that over the course of completing eight surveys, participants became accustomed to highlighting an answer without careful and precise consideration. Furthermore, research shows that psychological stress measures do not necessarily equate with their physiological correlates, suggesting that questionnaire measures may not represent an accurate depiction of an individual's internal state. Future research should also examine physiological measures, such as heart rate and cortisol variability, in addition to psychological measures to detect whether discrepancies exist or whether the data complement each other. This may help obtain a more objective portrayal of stress and wellbeing levels and filter out possible practice effects.

Secondly, the precise time of day at which participants completed the questionnaires was not specified. Consequently, there was considerable variability in the time that participants chose to do so, which may have affected their responses. Furthermore, participants were not instructed to chew gum at particular times of the day. As the questionnaire asked participants to rate how they were currently feeling, their responses may not have been related to chewing gum or workload if, for example, they had chewed gum during their lecture at $8 \mathrm{am}$ but completed the second questionnaire much later in the day. Future research should measure participant's stress levels, and the amount of gum chewed at specific times throughout the day, via an electronic diary, in order to ensure that workload and gum are related to participants' perceptions of stress and wellbeing, and determine whether variations in their responses can be attributed to chewing or working at certain times [44].

Thirdly, the extent to which participants experienced a high and low workload was not sufficiently controlled. Whilst some participants had no work on a low workload day, and ten hours of work on a high workload day, the reality of the criteria meant that another participant had one lecture on a low workload day and two lectures in addition to half-an-hours written work on a high workload day. Similarly, this variation also occurred within participants, so that the number of lectures an individual attended on their high workload days was significantly different from one another. Unfortunately, due to resource limitations, the only participants available were psychology students who attended a limited number of lectures per week. Also, the lectures that students were 
assigned to tended to be consistently spread out across the days of the week so that it was difficult to select days in which participants would have had a considerably higher workload than another. Future research should recruit students from different academic backgrounds, in which the difference between participants' workload is significantly large. Additionally, the study should involve some form of fixed criteria to satisfy a high and a low workload day.

Finally, limited measurement of workload was conducted. Future research should use the well-established measures of subjective workload. In addition, more attention should be paid to both short and longer term effects of the workload and the chewing gum intervention. Again, these are well established and could include physiological recording, measurement of cognitive performance, and electrophysiological measurement of brain function.

\section{Conclusion}

In conclusion, the present study showed that chewing a minimum of two pieces of gum for 20 minutes a day exerts beneficial effects on certain characteristics of perceived stress in students. This finding has promising implications; compared with some current forms of stress relief in students, and chewing gum may act as a cheaper and healthier alternative. Additionally, identifying that some qualities of perceived stress and wellbeing were reduced under a high workload, with or without chewing gum, may reassure students that a high workload is not necessarily detrimental to their wellbeing. This is likely to be of particular importance when approaching a deadline or during examination periods. Future research should now use a similar design over a longer intervention period to determine whether greater support could be obtained for the hypotheses. A thorough investigation into the physiological and psychological mechanisms responsible for the effects of chewing gum and workload on perceived stress and wellbeing will increase understanding of the processes involved in reducing stress, and help to maximize the benefits of chewing.

\section{References}

1. Reid, G.B., Nygren, T.E.: The Subjective Workload Assessment Technique: A Scaling Procedure for Measuring Mental Workload, vol. 52. North-Holland (1988)

2. Stassen, H.G., Johannsen, G., Moray, N.: Internal representation, internal model, human performance model and mental workload. Automatica 26(4), 811--820 (1990)

3. De Waard, D.: The measurement of drivers' mental workload. The Traffic Research Centre VSC, University of Groningen (1996)

4. Hart, S.G.: Nasa-task load index (nasa-tlx); 20 years later. In: Human Factors and Ergonomics Society Annual Meeting. vol. 50. Sage Journals (2006)

5. Smith, A.P., Smith, K.: Effects of workload and time of day on performance and mood. In: Megaw, E. D. (ed.) Contemporary Ergonomics, pp. 497--502. Taylor and Francis, London (1988)

6. Evans, M.S., Harborne, D., Smith A.P: Developing an objective indicator of fatigue: An alternative mobile version of the Psychomotor Vigilance Task (m-PVT). Presented at: H-WORKLOAD 2018: International Symposium on Human Mental Workload: Models and Applications, Amsterdam, The Netherlands, 20-21 September 2018. Longo, L., Leva, M.C. (eds.): H-WORKLOAD 2018, CCIS 1012, pp. 147--159, 2019. Springer Nature Switzerland. (2019)

7. Smith, A.P., Smith, H.N.: Workload, fatigue and performance in the rail industry. In: Longo, L., Leva M.C. (eds.) Human Mental Workload: Models and Applications. H-WORKLOAD 2017. Communications in Computer and Information Science, vol. 726, pp. 251-263. Springer, Cham. (2017)

8. Fan, J., Smith, A.P.: Mental workload and other causes of different types of fatigue in rail staff. In: Longo, L., Leva, M.C. (eds.) Human Mental Workload: Models and Applications. H-WORKLOAD 2018. Communications in Computer and Information Science 1012, pp. 147--159. Springer, Cham. (2019).

9. Cortes Torres, C.C., Sampei, K., Sato, M., Raskar, R., Miki, N.: Workload Assessment with Eye Movement Monitoring Aided by Non-invasive and Unobtrusive Micro-fabricated Optical Sensors. Adjunct Proceedings of the 28th Annual ACM Symposium on User Interface Software and Technology pp. 53--54 (2015).

10. Yoshida, Y., Ohwada, H., Mizoguchi, F., Iwasaki, H.: Classifying Cognitive Load and Driving Situation with Machine Learning. Int. J. Mach. Learn. Comput. 4(3), 210--215 (2014)

11. Wilson, G.F., Eggemeier, T.F.: Mental workload measurement. In: Karwowski, W. (ed.) International Encyclopedia of Ergonomics and Human Factors (2nd ed.), vol. 1, chap. 167. Taylor and Francis (2006)

12. Young, M.S., Stanton, N.A.: Mental workload. In: Stanton, N.A., Hedge, A., Brookhuis, K., Salas, E., Hendrick, H.W. (eds.) Handbook of Human Factors and Ergonomics Methods, chap. 39, pp. 1--9. CRC Press (2004) 
13. Young, M.S., Stanton, N.A.: Mental workload: Theory, measurement, and application. In: Karwowski, W. (ed.) International Encyclopedia of Ergonomics and Human Factors, vol. 1, pp. 818--821. Taylor and Francis, 2nd ed. (2006)

14. Moustafa, K., Saturnino, L., Longo, L.: Assessment of mental workload: a comparison of machine learning methods and subjective assessment techniques. In: 2017 1st International Symposium on Human Mental Workload: models and applications. vol. CCIS 726, pp. 30--50. Springer International Publishing (June 2017)

15. Hart, S.G., Staveland, L.E.: Development of NASA-TLX (Task Load Index): Results of Empirical and Theoretical Research. Adv. Psychol. 52(C), 139--183 (1988)

16. Tsang, P.S., Velazquez, V.L.: Diagnosticity and multidimensional subjective work- load ratings. Ergonomics. 39(3), 358--381 (1996)

17. Karasek Jr, R.A.: Job demands, job decision latitude, and mental strain: Implications for job redesign. Adm. Sci. Q. 285-308 (1979)

18. Longo, L., Leva M.C. (eds.) Human Mental Workload: Models and Applications. H-WORKLOAD 2017. Communications in Computer and Information Science, vol. 726, pp. 251-263. Springer, Cham. (2017)

19. Longo, L., Leva, M.C. (eds.) Human Mental Workload: Models and Applications. H-WORKLOAD 2018. Communications in Computer and Information Science. Springer, Cham. (2019).

20. Longo, L., Leva, M.C. (eds.) Human Mental Workload: Models and Applications. H-WORKLOAD 2019. Communications in Computer and Information Science. Springer, Cham. (2019).

21. Tosevski, D. L., Milovancevic, M. P.,Gajic, S.: Personality and psychopathology of university students. Current Opinion in Psychiatry, 23 (1), 48-52 (2010)

22. Dhabhar, F. S.: Acute stress enhances while chronic stress suppresses skin immunity: the role of stress hormones and leukocyte trafficking. Annals of the New York Academy of Sciences, 917, 876-893 (2000)

23. Atanackovic, D., Schnee, B., Schuch, G., Faltz, C., Schulze, J., Weber, C. S., Schafhausen, P., Bartels, K., Bokemeyer, C., Brunner-Weinzierl, M. C., Deter, H.C. (2006). Acute psychological stress alerts the adaptive immune response: Stress-induced mobilization of effector T cells. Journal of Neuroimmunology, 176, 141$152(2006)$

24. McEwan, B. S.: The neurobiology of stress: from serendipity to clinical relevance. Brain Research, 886, 172-189 (2010)

25. Juster, R. P., McEwan, B. S., Lupien, S. J.: Allostatic load biomarkers of chronic stress and impact on health and cognition. Neuroscience and Biobehavioural Reviews, 35, 2-16 (2009)

26. Rawson, H. E., Bloomer, K., Kendall, A.: Stress, anxiety, depression, and physical illness in college students. The Journal of Genetic Psychology: Research and Theory on Human Development, 155 (3), 321 $33(1994)$

27. Davis, C.: Decade ends with record student numbers. Organization for Economic Co-operation and Development. https://community.oecd.org/docs/DOC-4271 (2010)

28. Hamden-Mansour, A., Bandak, A., Puskar, K.: Effectiveness of cognitive- behavioural intervention on depressive symptomatology, stress and coping strategies among university students in Jordan. Issues in Mental Health Nursing, 30, 188-196 (2009)

29. Godbey, K. L., Courage, M. M.: Stress-management program: intervention in nursing student performance anxiety. Archives of Psychiatric Nursing, 8 (3), 190-199 (1994)

30. Saklofske, D. H., Austin, E. J., Mastoras, S. M., Beaton, L., Osborne, S. E.: Relationships of personality, affect, emotional intelligence and coping with student stress and academic success: Different patterns of association for stress and success. Learning and Individual Differences, 22 (2), 251-257 (2011)

31. Grossman, P., Niemann, L., Schmidt, S., Walach, H.: Mindfulness-based stress reduction and health benefits: A meta-analysis. Journal of Psychosomatic Research, 57 (1), 35-43 (2004)

32. Morris, S., Ford, L.: Universities 'can't cope with depressed students'. http://www.guardian.co.uk/society/2004/jun/18/mentalhealth.studenthealth (2004)

33. Princeton Review and Wrigley. Study habits survey. Princeton Review. New York (2005)

34. Ham, L. S., Hope, D. A.: College students and problematic drinking: A review of the literature. Clinical Psychology Review, 23 (5), 719-759 (2003)

35. Magid, V., Colder, C. R., Stroud, L. R., Nichter, M.: Negative affect, stress, and smoking in college students: unique associations independent of alcohol and marijuana use. Addictive Behaviours, 34 (11), 973 975 (2009)

36. University of Michigan Health System: Stress may lead students to use stimulants. http://www.sciencedaily.com•/releases/2008/04/080407195349.htm (2008)

37. Hollingworth, H. L.: Chewing as a technique of relaxation. Science, 90, 385-387 (1939)

38. Freeman, G. L.: Dr. Hollingworth on chewing as a technique of relaxation. Psychological Review, 47 (6). 491-493 (1940) 
39. Allen, A. P., Smith, A. P.: A review of the evidence that chewing gum affects stress, alertness and cognition. Journal of Behavioural and Neuroscience Research, 9, 7-23 (2011)

40. Smith, A. P.: Chewing gum, stress and health. Stress and Health, 25, 445-451 (2009)

41.Zibell, S., Madansky, E. (2009). Impact of gum chewing on stress levels. Online self- perception research study. Current Medical Research and Opinion, 25 (6), 1491-1500 (2009)

42. Allen, A.P., Smith, A. P.: Demand characteristics, pre-test attitudes and time-on-task trends in the effects of chewing gum on attention and reported mood in healthy volunteers. Appetite, 59, 349-356 (2011)

43. Smith, A. P., Chaplin, K., Wadsworth, E.: Chewing gum, occupational stress, work performance and wellbeing. An intervention study. Appetite, 58 (3), 1083-1086 (2012)

44. Allen, A.P., Smith, A.P. A brief intervention method for investigating the effects of chewing gum on occupational wellbeing. In M. Anderson (Ed.), Contemporary Ergonomics and Human Factors. Oxford, UK: Taylor and Francis (2012)

45. Smith, A. P., Woods, M. Effects of chewing gum on stress and work of university students. Appetite, 58, 1037-1040 (2012)

46. Smith, A. P.: Effects of chewing gum on cognition function, mood and physiology in stressed and nonstressed volunteers. Nutritional Neuroscience, 13 (1), 7-16 (2010)

47. Williams, G., Smith. A. P.: A holistic approach to stress and wellbeing. Part 6: The Wellbeing Process Questionnaire (WPQ short-form). Occupational Health (at Work) (2012)

48. Williams, G.: Developing short, practical measures of wellbeing. In M. Anderson (Ed.), Contemporary Ergonomics and Human Factors. Oxford, UK: Taylor and Francis (2012) 


\section{Appendix A}

\section{$\underline{\text { Instructions }}$}

GUM: HIGH, LOW. NO GUM: LOW, HIGH

Thank you for agreeing to participate in this project.

The aim of the experiment is to see whether chewing gum has an effect upon stress levels in students under a high and low workload.

Now for your part:

1. Please select 2 periods of 6 days within the next 2 weeks.

2. In the first 6 day period please select 1 day in which you have a high workload and another day in which you have a low workload, in that order. Please chew at least 2 pieces of gum on these 2 days for at least 20 minutes in total.

3. In the second 6 day period please select 1 day in which you have a low workload and another day in which you have a high workload, in that order. Please refrain from chewing gum on these 2 days.

4. High workload is defined as: a day in which you have two or more lectures and are working on an assignment and/or in paid/voluntary work. Low workload is defined as a day where you have one or less lectures and/or are not working on an assignment or in paid/voluntary work.

5. You need to complete this twice on each day: both at the beginning of each of the 4 days that you have chosen, and at the end of each day. 


\section{Appendix B}

\section{Gum survey}

Below is a list of descriptions that may or may not apply to you. For each description, please say to what extent you are experiencing this as you complete the questionnaire. Do not think for too long before answering but give your immediate reaction. Please be careful not to miss out any of the items. Give your answer by circling any number from 1 to 7 below the item, where appropriate.

$$
1=\text { not at all, } 4=\text { moderately and } 7=\text { extremely }
$$

1. Feeling anxious.

$\begin{array}{lllllll}1 & 2 & 3 & 4 & 5 & 6 & 7\end{array}$

2. Feeling depressed.

$\begin{array}{lllllll}1 & 2 & 3 & 4 & 5 & 6 & 7\end{array}$

3. Feeling stressed.

$\begin{array}{lllllll}1 & 2 & 3 & 4 & 5 & 6 & 7\end{array}$

4. Feeling mentally fatigued.

$\begin{array}{lllllll}1 & 2 & 3 & 4 & 5 & 6 & 7\end{array}$

5. Feeling physically fatigued.

$\begin{array}{lllllll}1 & 2 & 3 & 4 & 5 & 6 & 7\end{array}$

6. Having problems of memory, attention or action.

$\begin{array}{lllllll}1 & 2 & 3 & 4 & 5 & 6 & 7\end{array}$

7. Not getting as much work done as you would like.

$\begin{array}{lllllll}1 & 2 & 3 & 4 & 5 & 6 & 7\end{array}$

8. How many pieces of gum have you chewed today? Number of pieces:

9. How many hours of lectures have you attended today? Number of hours:

10. How many hours have you spent studying and/or in paid or voluntary work? Number of hours: 